\title{
Application of Time-Frequency Domain Reflectometry for Detection and Localization of a Fault on a Coaxial Cable
}

\author{
Yong-June Shin, Member, IEEE, Edward J. Powers, Life Fellow, IEEE, Tok-Son Choe, Student Member, IEEE, \\ Chan-Young Hong, Student Member, IEEE, Eun-Seok Song, Student Member, IEEE, \\ Jong-Gwan Yook, Senior Member, IEEE, and Jin Bae Park, Senior Member, IEEE
}

\begin{abstract}
In this paper, we introduce a new high-resolution reflectometry technique that operates simultaneously in both the time and frequency domains. The approach rests upon timefrequency signal analysis and utilizes a chirp signal multiplied by a Gaussian time envelope. The Gaussian envelope provides time localization, while the chirp allows one to excite the system under test with a swept sinewave covering a frequency band of interest. This latter capability is of particular interest when testing communication cables and systems. Sensitivity in detecting the reflected signal is provided by a time-frequency cross-correlation function. The approach is verified by experimentally locating various types of faults, located at various distances, in RG 142 and RG 400 coaxial cables.
\end{abstract}

Index Terms-Chirp signal, fault detection, fault location, resolution, time-frequency cross-correlation function, time-frequency domain reflectometry (TFDR).

\section{INTRODUCTION}

$\mathbf{T}$ HE importance of aging electrical wiring and associated faults in aircraft has been highlighted in [1]. This problem is not limited to aircraft only but also includes systems where complicated wiring is involved and high safety is required such as the space shuttle, nuclear power plants and very tall buildings [2]. Thus, the detection and localization of faults with high accuracy is required for diagnosis and maintenance of wiring systems.

The state-of-art for wiring/cable fault detection can be categorized by time domain analysis and frequency domain analysis. In time domain analysis, time domain reflectometry (TDR) [3] is used, whereas in frequency domain analysis, frequency domain reflectometry (FDR) [4] and standing wave reflectometry

Manuscript received June 15, 2003; revised January 31, 2005. This work was supported in by the Ministry of Commerce, Industry and Energy, Republic of Korea, Project 00015071, "Smart Wiring Optimal Signal Design and System Development for Electrical and Electronic Wiring Diagnosis," by the National Radio Research Laboratory, Seoul, Korea, and in part by the U.S. ONR under Grants N00014-02-1-0623 and N00014-00-0368.

Y.-J. Shin is with Department of Electrical Engineering, The University of South Carolina, Columbia, SC 29208 USA (e-mail: shinjune@engr.sc.edu).

E. J. Powers is with the Department of Electrical and Computer Engineering, The University of Texas, Austin, TX 78712 USA (e-mail: ejpowers@ mail.utexas.edu).

T.-S. Choe is with the Agency for Defence Development, Daejeon, Korea.

C.-Y. Hong and E.-S. Song are with Samsung Electronics, Suwon, Korea.

J.-G. Yook and J. B. Park are with the Department of Electrical and Electronics Engineering, Yonsei University, Seoul 120-749 Korea, (e-mail: tschoe@ yonsei.ac.kr)

Digital Object Identifier 10.1109/TIM.2005.858115
(SWR) [5] are utilized. Each methodology is based on the appropriate analysis of the reference signal and reflected signal either in the time or frequency domain only. However, in this paper, detection and location of coaxial cable faults by the use of joint time-frequency analysis is discussed.

TDR [6] and FDR are well-known conventional methods that have been applied to various types of applications including the cable test [7], network analyzer [8], and measurement of characteristic impedance [9], [10], etc. Recently, modern TDR instruments have been used for failure analysis and signal integrity characterization of high speed circuit boards [11], wiring packages, sockets, connectors, cable interconnects, and even power electronic devices [12], etc. However, the resolution and accuracy of the TDR and FDR are limited by the rise/fall time and frequency sweep bandwidth, respectively. To enhance the accuracy in reflectometry, digital signal processing techniques, e.g., the "cepstrum," has been suggested for TDR [13] and "cisoids" have been applied to FDR [14]. Recently, the problem of TDR waveform distortion has been investigated by the approximation of the skin effect to enhance accurate localization of a fault in cables [15]. However, the distortion of the TDR pulse is an inherent problem in TDR for accurate detection and localization of a fault.

In this paper, we introduce a joint time-frequency domain reflectometry (TFDR) technique which captures many of the advantages of TDR and FDR aforementioned. The reference signal is a (linear with time) chirp signal which allows one to apply the RF power in the band of interest. To provide time localization, the chirp signal is multiplied by a Gaussian envelope in the time domain. The time-frequency distributions [16] of the reference signal and the reflected signals are calculated. Then these two time-frequency distributions are cross correlated in the time-frequency domain. The peak in the time-frequency cross-correlation function allows one to estimate an accurate round-trip propagation time and, hence, distance, as in classical TDR. Yet, the experiment is carried in an RF band of interest which is relevant for the particular wire/cable under test, as in FDR. If frequency-dependent phenomena, such as dispersion, lead to substantial distortion of the reflected signal, these effects can be mitigated by reducing the frequency bandwidth of the reference signal.

In Section II, the fundamental idea of TFDR is discussed. The proposed signal design is based on the Gaussian envelope chirp signal. This signal is characterized by four parameters: 


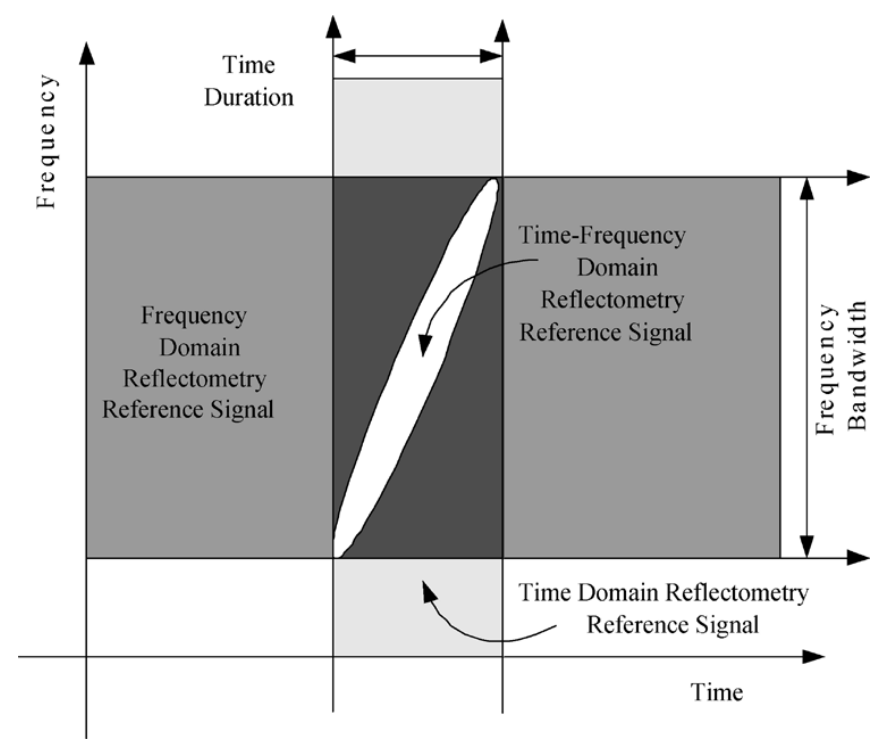

Fig. 1. Schematic comparison of TDR, FDR, and TFDR.

time duration, frequency sweep rate, center frequency, and time center. Next the time-frequency distributions of the reference and reflected signals are given. To detect and localize the fault using TFDR, the time-frequency cross-correlation function of the time-frequency distributions of the reference and reflected signal is introduced. To verify and demonstrate the postulated methodology, a set of 48 laboratory experiments is described in Section III. Twenty-four TFDR experiments were carried out using two types of coaxial cable (RG 142 and RG 400), three types of faults ("open," "short," and "damage") and four fault locations $(10,20,30$, and $40 \mathrm{~m})$. The 24 experiments were then repeated (making a grand total of 48) using a commercial TDR instrumentation for comparison purposes. The results are described in Section IV. For the same experimental conditions, the proposed TFDR resulted in somewhat better accuracy in fault localization than the TDR. These experimental results lead to the conclusion in Section V that TFDR is competitive with TDR with respect to fault localization, yet offers the additional advantage of placing RF energy in a frequency band of interest.

\section{TFDR}

In this section, the basic idea of TFDR is presented. To begin with, let us compare this new reflectometry methodology with traditional reflectometry methodologies. Fig. 1 schematically depicts reference signals for TDR, FDR, and TFDR on a timefrequency plane. As suggested in Fig. 1, TDR uses a pulse with fixed time duration and compares the reference and reflected signals in the time domain only. Therefore, TDR can not analyze the signal in the frequency domain because an ideal step pulse has its energy spread over a wide range of frequencies at the time instance of the step. On the other hand, FDR uses a set of sinusoidal signals with fixed frequency bandwidth and analyzes the change of the signal in the frequency domain only. As a result, it is difficult to analyze the signal in time domain by FDR because a pure sinusoidal signal, which is a reference signal in FDR, has, in principle, infinite time duration.
Therefore, instead of traditional TDR and FDR, we propose a new type of reflectometry, TFDR. TFDR uses a linearly modulated chirp signal with a Gaussian envelope. The proposed reference signal is written as follows:

$$
s(t)=\left(\frac{\alpha}{\pi}\right)^{1 / 4} e^{-\alpha\left(t-t_{0}\right)^{2} / 2+j \beta\left(t-t_{0}\right)^{2} / 2+j \omega_{0}\left(t-t_{0}\right)}
$$

where $\alpha, \beta, t_{0}$, and $\omega_{0}$ determine the time duration, frequency sweep rate, time center, and frequency center, respectively. The Gaussian envelope localizes the reference signal in the time and frequency domain while the instantaneous frequency of the signal increases with time in a linear manner as depicted in Fig. 1.

The appropriate determination of the four signal parameters for a specific application is very important in TFDR. The design of the reference signal that fits the physical characteristics of the RG type coaxial cable used in the experiments and post processing of the reflected signal for detection and localization will be addressed in the next subsections.

\section{A. Design of Reference Signal}

The central idea of TFDR is to design a reference signal that "fits" the physical characteristics of the target wire or cable in the time and frequency domain. Consider the proposed reference signal in (1). In (2)-(6) we describe several properties of this signal given in [16]. For this signal, one can evaluate the time center $\left(t_{s}\right)$ and the time duration $\left(T_{s}\right)$ as follows:

$$
\begin{aligned}
t_{s} & =\int t|s(t)|^{2} d t=t_{0}, \\
T_{s}^{2} & =\int\left(t-t_{s}\right)^{2}|s(t)|^{2} d t=\frac{1}{2 \alpha} .
\end{aligned}
$$

For convenience of calculation, assume that $t_{0}=0$.

The Fourier transform of the chirp signal in (1) is obtained as follows:

$$
S(\omega)=\sqrt{\frac{\sqrt{\alpha}}{\sqrt{\pi}(\alpha-j \beta)}} e^{-\left(\omega-\omega_{0}\right)^{2} / 2(\alpha-j \beta)} .
$$

Similarly, the frequency center $\left(\omega_{s}\right)$ and bandwidth $\left(B_{s}\right)$ can be evaluated in terms of $S(\omega)$ as follows:

$$
\begin{aligned}
\omega_{s} & =\int \omega|S(\omega)|^{2} d \omega=\omega_{0}, \\
B_{s}^{2} & =\int\left(\omega-\omega_{s}\right)^{2}|S(\omega)|^{2} d \omega=\frac{\alpha^{2}+\beta^{2}}{2 \alpha} .
\end{aligned}
$$

Consider the Wigner time-frequency distribution of the time signal $s(t)$ obtained by following transformation:

$$
W(t, \omega)=\frac{1}{2 \pi} \int s^{*}\left(t-\frac{1}{2} \tau\right) s\left(t+\frac{1}{2} \tau\right) e^{-j \tau \omega} d \tau .
$$

Then the Wigner distribution of the reference signal $W_{s}(t, \omega)$ is

$$
W_{s}(t, \omega)=\frac{1}{\pi} e^{-\alpha\left(t-t_{0}\right)^{2}-\left(\omega-\beta\left(t-t_{0}\right)-\omega_{0}\right)^{2} / \alpha} .
$$




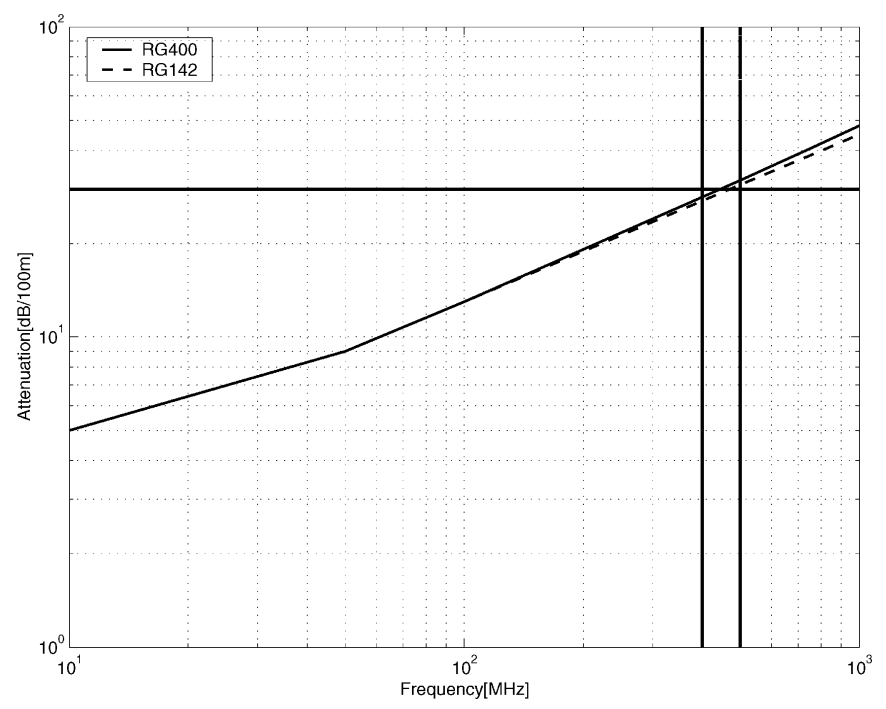

Fig. 2. Frequency-dependent attenuation characteristics of RG 142 (dotted line) and RG 400 (solid line) type coaxial cables in $\mathrm{dB}$ per $100 \mathrm{~m}$. The frequency region of interest is indicated by the two solid vertical lines: $400 \mathrm{MHz} \sim$ $500 \mathrm{MHz}$.

Equation (6) indicates how the energy in the reference signal is distributed over the time and frequency plane as indicated schematically in Fig. 1.

In order to apply the reference signal to the coaxial cables used in the experiment mentioned in the Introduction and Section III, the parameters of the signal are to be selected with consideration of the attenuation characteristics of the coaxial cables. The selection of the parameters take the following order:

\section{- step 1. Determine center frequency : \\ $\left(\omega_{0}\right)$ \\ - Step 2. Determine frequency bandwidth : $\left(B_{s}\right)$ \\ - step 3. Determine time duration : $\left(T_{s}\right)$}

Step 1. Center Frequency: The center frequency of the reference signal determines the degree of attenuation of the reference signal through the cable, since attenuation increases with frequency. Thus, one must consider a trade off, since higher frequencies allow higher spatial resolution with shorter wavelengths, but suffer more severe attenuation. Considering the noise sensitivity of the experimental setup, we find we can tolerate a round-trip attenuation of $24 \mathrm{~dB}$. The maximum fault distance in this set of experiments is $40 \mathrm{~m}$, so the maximum signal attenuation is $24 \mathrm{~dB} / 80 \mathrm{~m}$ or $30 \mathrm{~dB} / 100 \mathrm{~m}$. From Fig. 2, we see that the horizontal line of $30 \mathrm{~dB} / 100 \mathrm{~m}$ intersects the attenuation curves at approximately, $450 \mathrm{MHz}$, which we then select as our center frequency of the reference signal. Obviously, in the general case, the procedure used to select the center frequency will depend upon the the attenuation characteristics of the system under test, or possibly other RF characteristics of interest.

Step 2. Frequency Bandwidth: The experimental frequency bandwidth of the chirp signal generation is limited to the performance of the signal generator and circulator $(100 \mathrm{MHz}$ in this paper) which isolates the signal reflected from the fault. As a

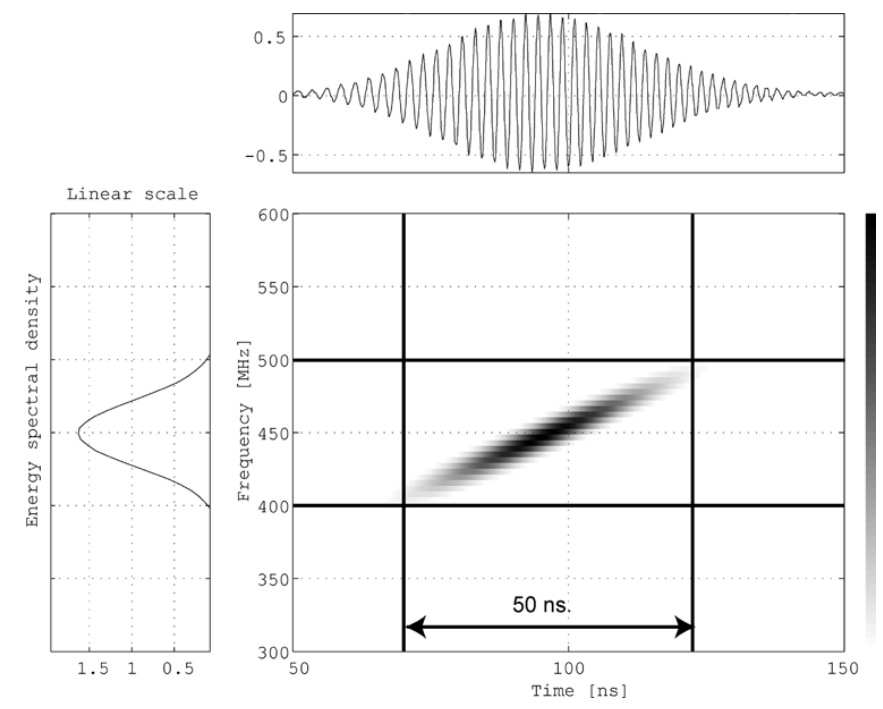

Fig. 3. Time-frequency distribution of the designed reference signal for RG type coaxial cables. The top plot is the reference signal time waveform, and the left hand plot is its corresponding energy spectrum.

result, the frequency bandwidth of the chirp signal is limited by $100 \mathrm{MHz}$ so that the frequency range of interest is selected as $400 \mathrm{MHz} \sim 500 \mathrm{MHz}$ as indicated in Fig. 2.

Step 3. Time Duration: The performance of the signal generator in terms of rise/fall time and frequency sweep capacity limit is $25 \mathrm{~ns}$. in this paper. In order to meet the parameters (center frequency and frequency bandwidth) selected above, the time duration of the reference signal is selected to be $50 \mathrm{~ns}$. We note that in time-frequency analysis the uncertainty principle places a constraint on the best time and frequency localization that can be achieved simultaneously. Observe that the product of frequency bandwidth $B_{s}$ and time duration $T_{s}$ in this experiment is equal to $10 \pi$ and therefore does not violate the uncertainty principle stated in [16].

In summary, the following parameters of the chirp signal (1) will be applied to the coaxial cable as a reference signal.

- $\quad$ Center Frequency : $\omega_{0} / 2 \pi=450 \mathrm{MHz}$

- $\quad$ Time Duration of Chirp : 50 ns.

- Frequency Bandwidth : $100 \mathrm{MHz}(400 \mathrm{MHz}$ $500 \mathrm{MHz})$

- Frequency Sweep : Linear increasing $(\beta / 2 \pi=$ $100 \mathrm{MHz} / 50 \mathrm{~ns}$ )

In Fig. 3, the designed time-frequency distribution of the experimental reference signal for RG type coaxial cable is provided. Also shown at the top is the time waveform of the Gaussian chirp signal and on the left its energy spectrum. As shown in Fig. 3, the reference signal provides time localization within $50 \mathrm{~ns}$ while the frequency is also localized between 400 and $500 \mathrm{MHz}$.

In this paper, the design of the reference signal is customized for RG type coaxial cables up to 40 meters in length, however, for longer distance testing, the time duration of the signal is to be increased while the center frequency is to be decreased so that the reference signal suffers less attenuation by the wire or cable under test. If a longer testing distance is required, one can design the reference signal in time-frequency domain by following the reference signal design procedures. The flexibility 
in designing a reference signal in TFDR also allows one to minimize frequency-dependent distortion of the reflected signal by assigning a relatively narrower frequency bandwidth.

\section{B. Detection and Localization by Time-Frequency Cross-Correlation Function}

For detection and localization of a fault in RG type coaxial cable, it is necessary to model the propagation of the reference signal in the media which allows one to determine the properties of the signal reflected from the fault in the coaxial cable.

Consider the spatial propagation and reflection of the timefrequency domain reference signal. As the signal propagates along the media with spatial variable $x$, the waveform will be changed by the transfer function of the media $H(\omega, x)$. For convenience of calculation, let the time center of the reference signal be $t_{0}=0$ without loss of generality. Let $u(x, t)$ be a waveform that is observed at a distance, $x$, for a given initial condition, $u(x=0, t)=s(t)$, then, the general solution of the $u(x, t)$ is,

$$
u(x, t)=\frac{1}{\sqrt{2 \pi}} \int S(\omega, x) e^{j \omega t} d \omega
$$

where

$S(\omega, x)=S(\omega, x=0) \cdot H(\omega, x)=S(\omega, x=0) e^{-(\alpha(\omega)-j k(\omega)) x}$

and where $H(\omega, x)$ is the transfer function of the medium which is characterized by the frequency-dependent attenuation $\alpha(\omega)$ and wave number $k(\omega)$ [17]. Note that the frequency-dependent attenuation $\alpha(\omega)$ is not to be confused with the parameter $\alpha$ in (1). In the following we assume linear frequency-dependent attenuation, $\alpha(\omega) \simeq A \omega$, and dispersion, $k(\omega) \simeq K \omega$. For the latter case, note that both the phase $(\omega / k)$ and group velocities $(\partial \omega / \partial k)$ are both equal to $1 / K$ and will, henceforth, be simply denoted by $v$, the velocity of propagation. The assumption of a dispersionless media is particularly suitable for the coaxial cable experiments considered later in this paper.

For the detection of the fault, the correlation of the time-frequency distribution of the the reference signal and reflected signal is utilized. Denote the reflected signal as $r(t)$ and its Wigner distribution as $W_{r}(t, \omega)$, and $W_{s}(t, \omega)$ as the Wigner distribution of the reference signal $s(t)$. Then one can evaluate a time-frequency cross-correlation function $C_{s r}(t)$ as follows:

$$
C_{s r}(t)=\frac{2 \pi}{E_{s} E_{r}} \iint W_{r}\left(t^{\prime}, \omega\right) W_{s}\left(t^{\prime}-t, \omega\right) d \omega d t^{\prime}
$$

where

$$
\begin{aligned}
& E_{r}=\iint W_{r}\left(t^{\prime}, \omega\right) d \omega d t^{\prime} \\
& E_{s}=\iint W_{s}(t, \omega) d t d \omega
\end{aligned}
$$

where the time integrals are carried out over the duration of the reference signal or reflected signal, as appropriate. The denominators $E_{r}$ and $E_{s}$ play the role of normalization factors so that the time-frequency cross-correlation function is bounded between 0 and 1.

Considering the transfer function of the coaxial cable, the time-frequency distribution of the reference signal is given by

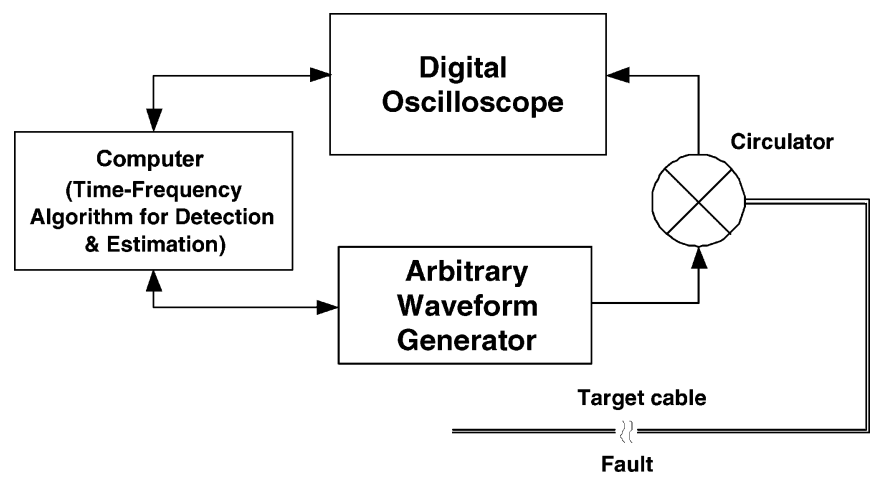

Fig. 4. Experimental setup for TFDR.

(6) and that of the reflected signal $W_{r}(t, \omega)$ can be obtained as follows:

$$
W_{r}(t, \omega)=\frac{1}{\pi} e^{-\alpha(t-x / v)^{2}-\left(\omega-\beta(t-x / v)-\omega_{0}\right)^{2} / \alpha} \cdot e^{-2 A x \omega} .
$$

Then the time-frequency cross-correlation function of the reflected signal $C_{s r}(t)$ is

$$
C_{s r}(t)=e^{-\left(\left(\alpha^{2}+\beta^{2}\right) / 2 \alpha\right)(A x)^{2}} \cdot e^{-\left(\left(\alpha^{2}+\beta^{2}\right) / 2 \alpha\right)(t-x / v)^{2}} .
$$

Therefore, the existence of the reflected signal is to be detected by a quantitative number between 0 and 1 . From examination of the time-frequency cross-correlation function $C_{s r}(t)$ in (13), it is clear that it will be a maximum (local peak time) at $t=x / v$, as expected. The first term on the RHS decreases because, as the reflected signal travels farther, its high frequency content will be attenuated more, and thus the reflected signal will be less correlated with the reference signal.

In Sections III and IV, the local peak time of the time-frequency cross-correlation function will be utilized to accurately measure the propagation delay of the reflected signal, which is then to be converted into the fault location with knowledge of the velocity of propagation.

\section{EXPERIMENTAL SETUP}

To demonstrate the ability of TFDR to detect and locate various types of faults in RG 142 and RG 400 type coaxial cables, an experimental TFDR system is organized as shown in Fig. 4. The system consists of a circulator, an arbitrary waveform generator (Tektronix, AWG 610) and an oscilloscope (Agilent Infinium) which are connected to a computer with GPIB cable for automatic control of the instruments. The computer controls the arbitrary waveform generator (AWG) to produce the Gaussian envelope chirp signal which propagates into the target cable via the circulator. This reference signal is reflected at the fault location and back to the circulator. The circulator redirects the reflected signal to the digital oscilloscope. The computer controls and synchronizes the arbitrary waveform generator and digital oscilloscope, calculates the time-frequency distribution of the reference signal and reflected signals, and executes the time-frequency cross-correlation algorithm.

The purpose of the experiment is to verify the feasibility of TFDR. Furthermore, the performance is to be compared with a commercial TDR. The experiment is carried out for two types of coaxial cables, i.e., RG 142 and RG 400 types. Various dis- 
(a)

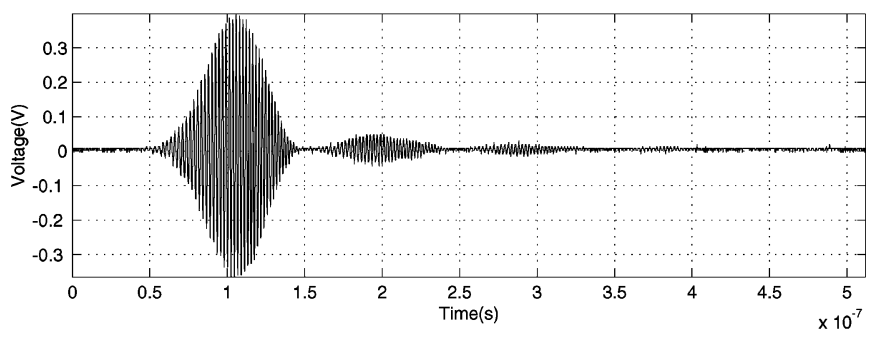

(b)

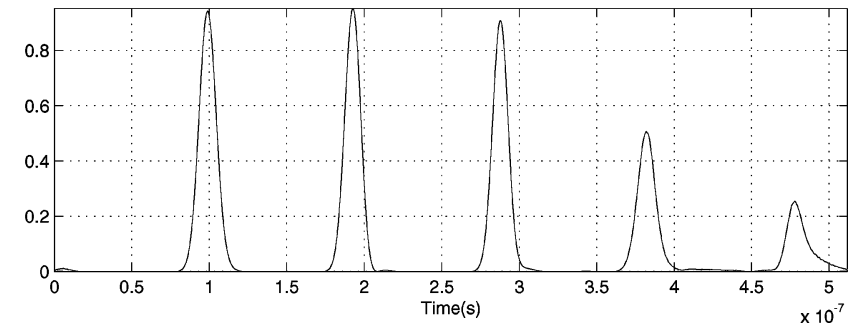

Fig. 5. Time series of the reflected signal in (a) and corresponding timefrequency cross-correlation function for detection and estimation of the "damage" fault in (b) for a fault at $10 \mathrm{~m}$ in RG 142 type coaxial cable. (The first peak of correlation is at $96.5 \mathrm{~ns}$. and the second one is at $191.0 \mathrm{~ns}$.).

tances and types of faults are considered. The actual location of the faults varies from $10,20,30$, and $40 \mathrm{~m}$ while the types of faults are classified as "open," "short," and "damage." The "damage" of the coaxial cable is emulated by a failure of the external shields so that the internal dielectric material is exposed over $1 \mathrm{~cm}$. Thus a total of 24 experiments ( 2 types of cables $\times 4$ fault distances $\times 3$ types of faults) are carried out. To evaluate the performance of TFDR in fault detection and localization, the same 24 experiments are repeated using a commercial TDR system which has a rise time of 300 ps.

\section{RESUlt AND ANALYSIS}

As indicated in Section III, we have carried out several experiments in which we compare the ability of TFDR and TDR to locate various types of faults at various distances in coaxial cable. For a fair comparison between TFDR and TDR, we use the same velocity of propagation, $v=2.10 \times 10^{8} \mathrm{~m} / \mathrm{sec}$, which was obtained experimentally for both types of cables. Also note that the sampling rates for reflected signal acquisition of the TFDR is $4 \mathrm{GHz}$ while that of the TDR is $12 \mathrm{GHz}$ in this experiment.

\section{A. Experimental Result}

In Fig. 5, we consider the case of RG 142 type coaxial cable which has a damage-type fault located 10 meters away from the source of the reference signal. Fig. 5(a) is the acquired time series of the reflected signal at a $4-\mathrm{GHz}$ sampling rate while Fig. 5(b) is the time-frequency cross correlation between the time-frequency distributions of the reference signal and the reflected signal. In Fig. 5(a) and (b), the reference signal and the first reflected signal from the fault are observed around 100 and $190 \mathrm{~ns}$, respectively. The remaining peaks in the timefrequency cross correlation are caused by multiple reflections. Note, however, as shown in Fig. 5, the waveform caused by multiple reflections can be accurately detected and localized by the time-frequency cross-correlation function, while the multiple reflections are a potential problem in TDR. (a)

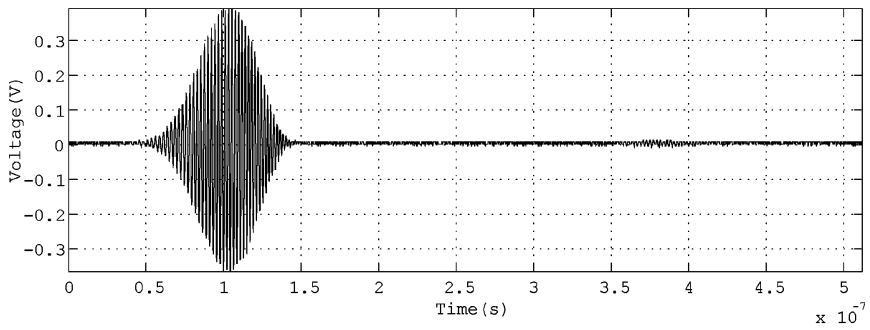

(b)

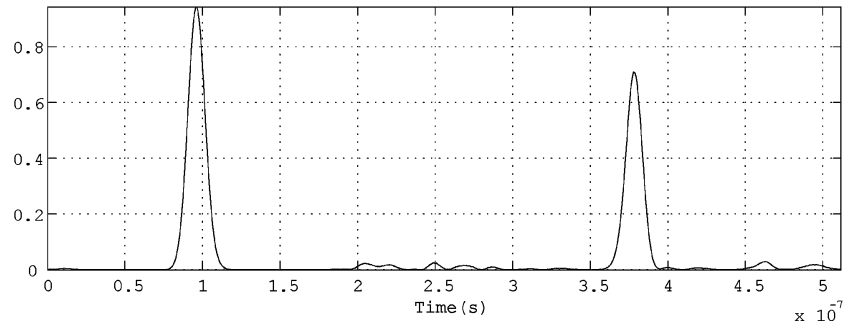

Fig. 6. Time series of the reflected signal in (a) and corresponding timefrequency cross-correlation function for detection and estimation of the "damage" fault in (b) for a fault at $30 \mathrm{~m}$ in RG 400 type coaxial cable. (The first peak of correlation is at $96.3 \mathrm{~ns}$. and the second one is at $379.7 \mathrm{~ns}$.).

The time-frequency cross correlation provided in Fig. 5(b) can be interpreted via (13): with increasing propagation distance $x$, the peak value of the time-frequency correlation function exponentially decays for the reason described previously, while the time spread of the time-frequency correlation function is inversely proportional to the frequency bandwidth of the reference signal as indicated in (4) and (13). The second local peak of the time-frequency cross-correlation function indicates the presence of the reflected signal which enables one to determine the round-trip propagation time. The estimated time of the first peak, which is the reference signal itself is $96.5 \mathrm{~ns}$, while the estimated time of the second peak is $191.0 \mathrm{~ns}$. With the obtained $v$ of the RG 142 coaxial cable $\left(2.10 \times 10^{8} \mathrm{~m} / \mathrm{s}\right)$, the location of the fault can be estimated to be $9.92 \mathrm{~m}$.

The example provided in Fig. 6 presents a sample case for RG 400 type coaxial cable which has a damage-type fault located $30 \mathrm{~m}$ away from the source of the reference signal. In Fig. 6(a), the signal that occupies the time duration between 50 and $150 \mathrm{~ns}$ is the reference signal, and the signal component located in the time interval between 350 and $400 \mathrm{~ns}$ (which is difficult to see in the time domain) is the reflected signal from the damage to the cable. Fig. 6(b) is the time-frequency cross correlation between the reference signal and the reflected signal time-frequency distributions: the first peak (estimated time : $96.3 \mathrm{~ns}$ ) of the correlation function in Fig. 6(b) reveals the existence of the reference signal itself, and the second peak (estimated time: $379.7 \mathrm{~ns}$ ) indicates the presence of a fault. The location of the fault is evaluated to be $29.76 \mathrm{~m}$.

In order to compare the performance in accuracy between TDR and TFDR, the same coaxial cable with the same fault is tested using a commercial TDR instrument. Fig. 7 is acquired by the TDR instrument under the same conditions as the experiment corresponding to Fig. 6. TDR detects the fault through a step change of the reflected signal, and estimates the time of arrival from the time the step-change occurs. However, as shown in Fig. 7, the ideal step of the reference signal is smeared in the 


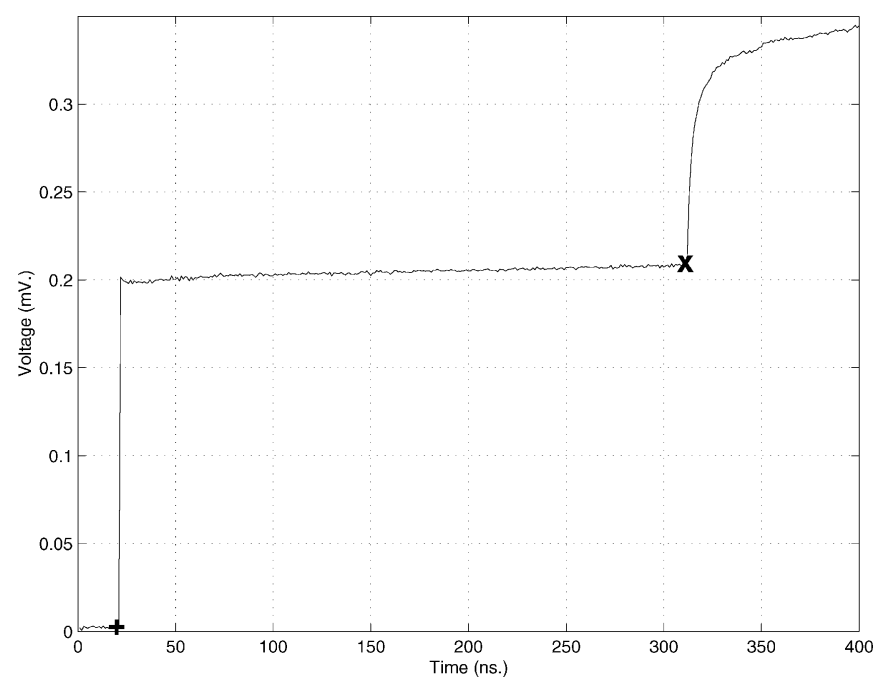

Fig. 7. A screen snapshot of the TDR for the detection and estimation of the fault location for a fault at $30 \mathrm{~m}$ in RG 400 type coaxial cable. (The first marker is at $23.5 \mathrm{~ns}$. and the second one is at $304.5 \mathrm{~ns}$.).

TABLE I

COMPARISON OF TDR AND TFDR ESTIMATES OF FAULT LOCATION FOR FOUR FAULT LOCATIONS, AND THREE TYPES OF FAULT FOR RG 142 TyPe COAXIAL CABLE

\begin{tabular}{|c|c||c|c||c|c|}
\hline & & $\begin{array}{c}\text { RG142 } \\
(\text { TFDR })\end{array}$ & $\begin{array}{c}\text { Error } \\
(\mathrm{m})\end{array}$ & $\begin{array}{c}\text { RG142 } \\
(\text { TDR })\end{array}$ & $\begin{array}{c}\text { Error } \\
(\mathrm{m})\end{array}$ \\
\hline \hline \multirow{3}{*}{$40 \mathrm{~m}$} & damage & 39.80 & 0.20 & 39.61 & 0.39 \\
\cline { 2 - 6 } & open & 39.78 & 0.22 & 39.47 & 0.53 \\
\cline { 2 - 6 } & short & 39.77 & 0.23 & 39.47 & 0.53 \\
\hline \multirow{3}{*}{$30 \mathrm{~m}$} & damage & 29.80 & 0.20 & 29.55 & 0.45 \\
\cline { 2 - 6 } & open & 29.78 & 0.22 & 29.55 & 0.45 \\
\cline { 2 - 6 } & short & 29.78 & 0.22 & 29.32 & 0.68 \\
\hline \multirow{3}{*}{$20 \mathrm{~m}$} & damage & 19.84 & 0.16 & 19.27 & 0.73 \\
\cline { 2 - 6 } & open & 19.84 & 0.16 & 19.56 & 0.44 \\
\cline { 2 - 6 } & short & 19.86 & 0.14 & 19.56 & 0.44 \\
\hline \multirow{3}{*}{$10 \mathrm{~m}$} & damage & 9.92 & 0.08 & 9.69 & 0.31 \\
\cline { 2 - 6 } & open & 9.88 & 0.12 & 9.67 & 0.33 \\
\cline { 2 - 6 } & short & 9.89 & 0.11 & 9.67 & 0.33 \\
\hline
\end{tabular}

reflected signal so that it is not always easy to measure accurately the arrival time of the reflected step. The location of the fault is measured to be $29.51 \mathrm{~m}$.

\section{B. Analysis and Discussion of Results}

To compare the fault location accuracy of TFDR and TDR, the location of the faults vary from $10,20,30$, to $40 \mathrm{~m}$. In each location, three types of faults are considered: "open," "short," and "damage" as described in Section III. The overall experimental results are summarized in Tables I and II for the RG 142 and RG 400 type coaxial cables, respectively. Note that the experimental results provided in the tables are obtained from several trials for each case. The TFDR experimental determination of propagation time was repeatable within to \pm 2 sample periods, which corresponds to $\pm 0.105 \mathrm{~m}$. As one can see in Tables I and II, TFDR yields a smaller error than TDR for all types and locations of the faults. The errors for TDR vary from 0.31 to $0.74 \mathrm{~m}$ while the errors of TFDR range from 0.08 to $0.28 \mathrm{~m}$, for all cases. Note that in TFDR, the detection and localization process is completely automated based on identifying the peaks of the time-frequency correlation function; however, the operation of the TDR involves adjusting
TABLE II

COMPARISON OF TDR AND TFDR ESTIMATES OF FAULT LOCATION FOR Four FAUlT LOCATIONS, AND THREE TyPES OF FAULT FOR RG 400 TyPe COAXIAL CABLE

\begin{tabular}{|c|c||c|c||c|c|}
\hline & & $\begin{array}{c}\text { RG400 } \\
(\text { TFDR })\end{array}$ & $\begin{array}{c}\text { Error } \\
(\mathrm{m})\end{array}$ & $\begin{array}{c}\text { RG400 } \\
(\text { TDR })\end{array}$ & $\begin{array}{c}\text { Error } \\
(\mathrm{m})\end{array}$ \\
\hline \hline \multirow{3}{*}{$40 \mathrm{~m}$} & damage & 39.74 & 0.26 & 39.37 & 0.63 \\
\cline { 2 - 6 } & open & 39.64 & 0.26 & 39.26 & 0.74 \\
\cline { 2 - 6 } & short & 39.85 & 0.15 & 39.37 & 0.63 \\
\hline \multirow{3}{*}{$30 \mathrm{~m}$} & damage & 29.76 & 0.24 & 29.51 & 0.49 \\
\cline { 2 - 6 } & open & 29.72 & 0.28 & 29.41 & 0.59 \\
\cline { 2 - 6 } & short & 29.77 & 0.23 & 29.48 & 0.52 \\
\hline \multirow{3}{*}{$20 \mathrm{~m}$} & damage & 19.90 & 0.10 & 19.58 & 0.42 \\
\cline { 2 - 6 } & open & 19.87 & 0.13 & 19.47 & 0.53 \\
\cline { 2 - 6 } & short & 19.87 & 0.13 & 19.58 & 0.42 \\
\hline \multirow{3}{*}{$10 \mathrm{~m}$} & damage & 9.91 & 0.09 & 9.51 & 0.49 \\
\cline { 2 - 6 } & open & 9.89 & 0.11 & 9.62 & 0.38 \\
\cline { 2 - 6 } & short & 9.90 & 0.10 & 9.62 & 0.38 \\
\hline
\end{tabular}

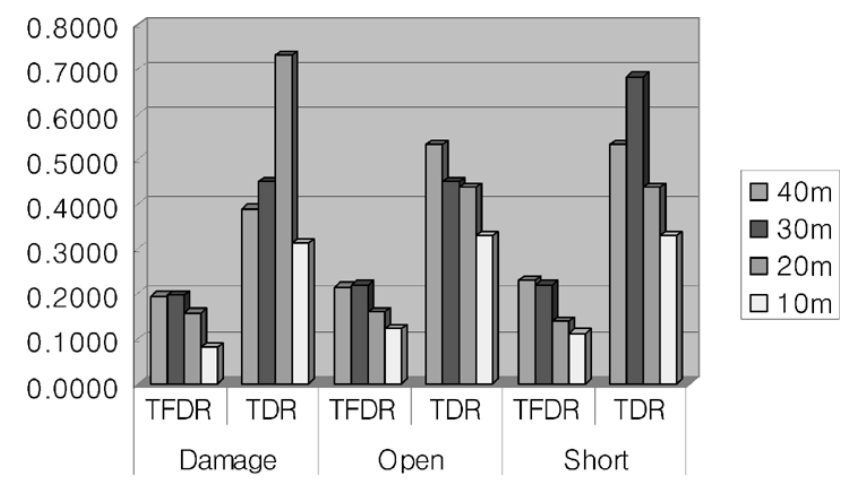

Fig. 8. Comparison of the fault location percentage error for TFDR and TDR for RG-142 coaxial cable for four different fault locations. (10, 20, 30, and $40 \mathrm{~m})$ and for three different faults (damage, open, and short).

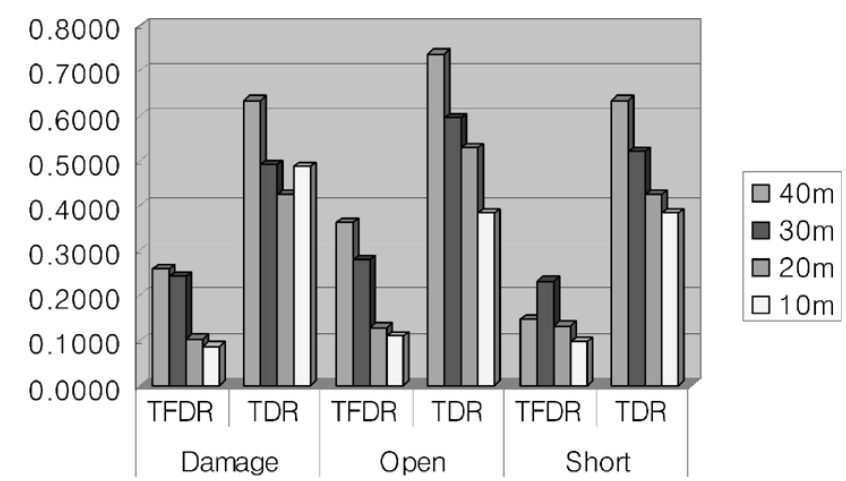

Fig. 9. Comparison of the fault location percentage error for TFDR and TDR for RG-400 coaxial cable for four different fault locations (10, 20, 30, and $40 \mathrm{~m}$ ) and for three different faults (damage, open, and short).

markers on the smeared edges in the reflected signal as shown in Fig. 7, and is thus subject to human judgement.

For a more graphical comparison of the results, the corresponding percentage error for each cable type is evaluated and plotted in Figs. 8 and 9. As one can see in these figures, TFDR yields a smaller percentage error than TDR for all locations of the fault: the errors for TDR are between $0.3 \%$ and $0.8 \%$ while the errors of TFDR are under $0.4 \%$ for all cases. Both TDR and TFDR show a tendency for the error rate to increase with distance. As the length of the cable increases, the energy of the reflected signal will decrease exponentially, which results in a lower SNR for both TDR and TFDR. The experimental results suggest that 
TFDR is capable of fault detection with a performance comparable to that of TDR. Yet the ability to design the reference signal for a particular cable or system being tested is a powerful advantage. To put it another way, TFDR possesses many of the advantages of TDR, while at the same time permitting one to consider the frequency-domain properties of the system under test. Thus, one possibility is to consider TFDR as being complementary to TDR in that TFDR is relatively easy to implement by adding an arbitrary waveform generator and digital signal processing unit to calculate the time-frequency cross-correlation function of the sampled reference and reflected waveforms.

\section{CONCLUSION}

In this paper, we introduce joint TFDR, which incorporates many of the advantages of time domain and frequency domain reflectometry. Faults on wires and cables are located from knowledge of the propagation time and velocity of propagation as in TDR. However, the use of a chirp signal with a Gaussian envelope enables one to effectively use a swept frequency reference signal as in FDR. This latter capability is very important when testing wires/cables used for communication purposes. The use of time-frequency cross-correlation function of the respective time-frequency distributions of the reference and reflected signals has proven to be a sensitive detector of weak reflected signals (see Fig. 6, for example). The experimental work involving two types of coaxial cable, three types of faults, and four fault lengths, indicates that the new TFDR locates faults with an accuracy comparable (actually, slightly better in our experiments) to TDR and, therefore, suggests that joint TFDR offers much promise as a new wire and cable diagnostic tool, especially in the communication area.

The contribution of the TFDR to the existing reflectometry field can be summarized as follows:

1) Accurate and sensitive detection and localization of the reflected signal via time-frequency cross correlation.

2) Flexibility of reference signal design depending on the physical characteristics of system under test. For example, one can place the RF energy in the frequency band of interest.

3) Successful detection and localization of multiple reflections, even very weak reflections.

4) Minimization of the distortion of the reference signal by limiting the frequency bandwidth, where necessary.

Clearly, additional work must be done to explore the promise of TFDR including: application of TFDR to faults in wiring other than coaxial cable; consideration of noise, particularly in the case of very weak reflected signals; consideration of the effects of dispersion where appropriate; the identification of those factors affecting the accuracy of fault location; and a detailed comparison of TFDR with TDR, FDR, and SWR in order to fully elucidate the advantages and limitations of TFDR.

\section{REFERENCES}

[1] C. Furse and R. Haupt, "Down to the wire: The hidden hazard of aging aircraft wiring," IEEE Spectrum, pp. 35-39, Feb. 2001.

[2] "Review of federal programs for wire system safety-final report," National Science and Technology Council Committee on Technology, Wire System Safety Interagency Working Group, 2000.
[3] C. P. Nemarich, "Time domain reflectometry liquid level sensors," IEEE Instrum. Meas. Mag., vol. 4, pp. 40-44, Dec. 2001.

[4] M. Yoshida, K. Nakamura, and H. Ito, "A new method for measurement of group velocity dispersion of optical fibers by using a frequencyshifted feedback fiber laser," IEEE Photon. Technol. Lett., vol. 13, pp. 227-229, Mar. 2001

[5] N. Kamdor and C. Furse, "An inexpensive distance measuring system for location of robotic vehicles," in Antennas and Propagation Society, IEEE Int. Symp. 1999, vol. 3, 1999, pp. 1498-1501.

[6] Hewlett Packard, Time Domain Reflectometry, Hewlett Packard, 1998.

[7] Application Guide. Riser Bond, Inc. [Online]. Available: http://www. riserbond.com/assets/downloads/Appguide.pdf

[8] H. Yamada, M. Ohmiya, Y. Ogawa, and K. Itoh, "Super resolution techniques for time-domain measurements with a network analyzer," IEEE Trans. Antennas Propag., vol. 39, pp. 177-183, Feb. 1991.

[9] N. G. Paulter, "An assessment on the accuracy of time-domain reflectometry for measuring the characteristic impedance of transmission line," IEEE Trans. Instrum. Meas., vol. 50, pp. 1381-1388, Oct. 2001.

[10] Agilent Technologies Impedance Measurement Handbook, 2nd ed., Agilent Technologies Co. Ltd., 2002.

[11] W. Kim, M. Swaminathan, and Y. Li, "Extraction of the frequencydependent characteristic impedance of transmission lines using TDR measurements," in IEEE Proc. Electronics Packaging Technology Conf., 2000, pp. 191-197.

[12] H. Zhu, A. R. Hefner Jr., and J.-S. (J.) Lai, "Characterization of power electronics system interconnect parasitics using time domain reflectometry," IEEE Trans. Power Electron., vol. 14, pp. 622-628, Jul. 1999.

[13] L. P. Van Biesen et al., "High accuracy location of faults on electrical lines using digital signal processing," IEEE Trans. Instrum. Meas., vol. 39, pp. 175-179, Feb. 1990.

[14] H. Vanhamme, "High resolution frequency-domain reflectometry," IEEE Trans. Instrum. Meas., vol. 39, pp. 369-375, Apr. 1990

[15] D. Agrez, "Approximation of the skin effect to improve cable-fault location by TDR," in Proc. IEEE Instrum. Meas. Conf. 2003, May 2003 , pp. 50-53.

[16] L. Cohen, "Time-frequency distributions - A review," Proc. IEEE, vol. 77, pp. 941-981, Jul. 1989.

[17] _ "Pulse propagation in dispersive media," in Proc. 10th IEEE Workshop Statistical Signal and Array Processing, 2000, pp. 485-489.

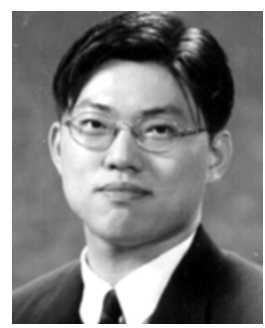

Yong-June Shin (M'00) received the B.S degree from Yonsei University, Seoul, Korea, in 1996 with early completion honors and the M.S degree from The University of Michigan, Ann Arbor, in 1997. He received the Ph.D. degree from the Department of Electrical and Computer Engineering, The University of Texas at Austin, in 2004.

Upon graduation, he joined the Department of Electrical Engineering, The University of South Carolina, Columbia, as an Assistant Professor. His research interests include time-frequency analysis, wavelets, and higher order statistical signal analysis. His fields of application are power systems, communications engineering, measurement and instrumentation, offshore technology, and biomedical engineering.

Dr. Shin is a member of Seoul Science High School Alumni Society.

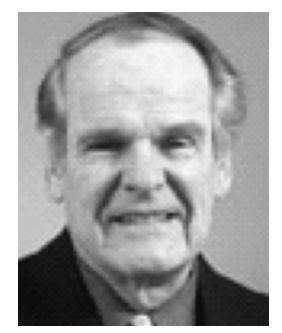

Edward J. Powers (S'57-M'58-SM'80-F'83LF'97) received the B.S. degree from Tufts University, the M.S. degree from the Massachusetts Institute of Technology, Cambridge, and the Ph.D. degree from Stanford University, Stanford, CA, in 1957, 1959, and 1965, respectively, all in electrical engineering.

$\mathrm{He}$ is currently the Texas Atomic Energy Research Foundation Professor in Engineering and past Director of the Telecommunications and Signal Processing Research Center at The University of Texas at Austin. His primary professional interests lie in the innovative application of digital higher-order statistical signal processing in the analysis, interpretation, and modeling of random data characterizing nonlinear physical phenomena and systems; and the utilization of the wavelet transform and time-frequency analysis to detect and identify transient events in various physical systems. 


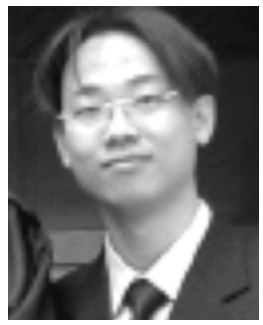

Tok-Son Choe (S'05) received the B.S. and M.S. degrees from Yonsei University, Seoul, Korea, in 2003 and 2004, respectively.

$\mathrm{He}$ is currently with the Agency for Defence Development, Daejeon, Korea. His interest fields of research are time-frequency analysis, robotics, and UWB radar.

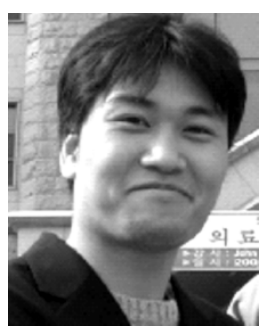

Chan-Young Hong (S'05) received the B.S. degree and the M.S. degree from Yonsei University, Seoul, Korea, in 2002 and 2004, respectively.

$\mathrm{He}$ is currently with Samsung Electronics, Suwon, Korea. His fields of research are time-frequency analysis, wavelets, robotics, and neural networks.

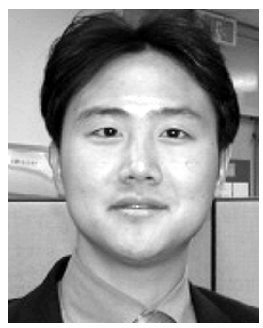

Eun-Seok Song (S'05) received the B.S. degree from Hongik University, Seoul, Korea, in 2001 and the M.S. degree from the Department of the Electrical and Electronic Engineering, Yonsei University, Seoul.

He is currently with the Interconnect and Product Technology Development Team, System LSI Division, Semiconductor Network Business, Samsung Electronics Co., Ltd., Suwon, Korea. His fields of research include EMI prediction, modeling of PDN and power integrity, signal processing, measurement and instrumentation, and computational electromagnetics.

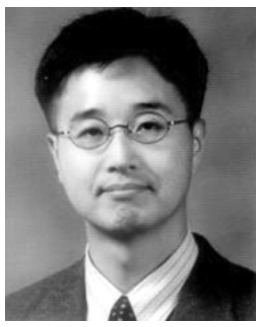

Jong-Gwan Yook (S'89-M'02-SM'04) received the B.S. and the M.S. degrees from Yonsei University, Seoul, Korea, and the Ph.D. degree from The University of Michigan, Ann Arbor, in 1987, 1989, and 1996, respectively, all in electrical engineering.

After receiving the Ph.D. degree, he worked for Qualcomm Inc., San Diego, CA, as a Senior Engineer from 1998 to 1999, and the Kwang-Joo Institute of Science and Technology from 1999 to 2000 as an Assistant Professor. He is currently an Associate Professor with the Department of Electrical and Electronic Engineering, Yonsei University, and Director of the Advanced Computational Electromagnetics Laboratory. His primary professional interests include microwave circuits and antennas, RF MEMS, computational electromagnetics, EMI/EMC analysis, and high-speed digital design and packaging.

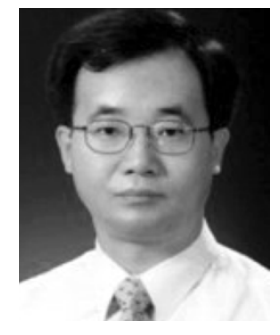

Jin Bae Park (SM'04) received the B.S. degree from Yonsei University, Seoul, Korea, and the M.S and $\mathrm{Ph} . \mathrm{D}$. degrees from Kansas State University, Manhattan, in 1977, 1985, and 1990, respectively, all in electrical engineering.

After receiving the Ph.D. degree, he was with the Department of Electrical and Computer Engineering, Kansas State University, from 1990 to 1991 as an Assistant Professor. He has been a professor with the Department of Electrical and Electronic Engineering, Yonsei University, since 1992 and he is currently a Director of the Control Engineering Laboratory and Automation Technology Research Center. His primary professional interests include measurement and instrumentation, optics, nonlinear control, robust control, fuzzy systems, neural network, signal processing, and robotics. 